\title{
Infectious Sacroiliitis: A Radiographic No Touch Lesion
}

\author{
Ashwin Malhotra ${ }^{1,2^{*}}$, Ramsey Kalil ${ }^{1}$, Ryan Jones ${ }^{1,3}$, Danielle Schwartz ${ }^{1}$, Asem H Qadeer ${ }^{1}$, Mingqian Huang ${ }^{4}$ and Robert Abdullah $^{5}$ \\ ${ }^{1}$ Department of Medicine, Stony Brook University Hospital, New York, USA \\ ${ }^{2}$ Department of Neurology, New York-Presbyterian Hospital/Weill Cornell Medical Center, New York, USA \\ ${ }^{3}$ Department of Neurology, Stony Brook University Hospital, New York, USA \\ ${ }^{4}$ Department of Radiology, Stony Brook University Hospital, New York, USA \\ ${ }^{5}$ Department of Medicine, Stony Brook University Hospital, Health Sciences Tower, New York, USA
}

*Corresponding author: Ashwin Malhotra, Department of Medicine, Stony Brook University Hospital, New York, USA, Tel: 516-749-4678; E-mail: asm9059@nyp.org Received date: October 14, 2017; Accepted date: October 20, 2017; Published date: October 23, 2017

Copyright: (c) 2017 Malhotra A, et al. This is an open-access article distributed under the terms of the Creative Commons Attribution License, which permits unrestricted use, distribution, and reproduction in any medium, provided the original author and source are credited.

Keywords: Hip pain; Magnetic resonance imaging; Sacroiliitis

\section{Introduction}

Infectious sacroiliitis (ISI) is relatively uncommon and accounts for $1-4 \%$ of all cases of septic arthritis [1]. Diagnosis can be challenging and is often delayed due to the wide spectrum of clinical presentations. Frequently, hip and buttock pain with often unrevealing plain radiographs of the pelvis and sacroiliac joints (SI joint) are the initial manifestation of the disease. A clinical exam including a FABER's test (flexion, abduction and external rotation) or the pelvic stress test, are approximability $70 \%$ sensitive [2].

As a result, the initial diagnosis of infectious sacroiliitis can easily be missed and result in complications such as abscess formation, disseminated infection, thromboembolism, late arthritis, and chronic debilitation, all of which require aggressive and invasive intervention. Magnetic resonance imaging (MRI) is the gold standard imaging modality for the diagnosis of sacroiliitis [2]. Here we present a challenging case that simultaneously underscores the noteworthy utility of MRI findings in diagnosing infectious sacroiliitis, and demonstrates the limitations of MRI in monitoring response to treatment.

\section{Case Presentation}

A previously healthy 46-year-old female presented to the emergency department (ED) with a one day history of left hip-buttock pain with mild radiation down the side of her thigh, but not extending to the knee. She denied paresthesias or any recent trauma and exhibited isolated left leg weakness secondary to pain. The patient endorsed that her pain was worse with movements and weight bearing. Radiographs of the pelvis and sacroiliac joints were unrevealing and the patient was discharged home with a diagnosis of sciatica and a prescription for a short course of oxycodone and methocarbamol.

Six days later, the patient presented again to the ED with inability to walk secondary to significantly worsened left hip-buttock pain. She was unable to move her leg without severe pain. She denied fevers, trauma, recent surgeries or illness.

The patient was found to be afebrile with labs significant for leukocytosis $(16,540$ cells/L) and elevated inflammatory markers (Erythrocyte Sedimentation Rate [ESR] 98 mm/hr, C-Reactive Protein [CRP] $28.2 \mathrm{mg} / \mathrm{dL}$ ).

Physical exam was significant for extremities with soft compressible compartments (no lesions, wounds or erythema); extreme left hip- buttock pain with log roll and axial load on left lower extremity; and no pain on right sided exam (still able to bear weight on the right side).

Straight leg raise evaluation was limited secondary to intense pain bilaterally. Motor and sensory functions were intact throughout other than left lower extremity where strength was diminished secondary to pain in proximal muscle groups with preserved dorsiflexion and plantarflexion.

On further history, the patient denied intravenous drug use, recent gynecological interventions, and history of infections such as cellulitis or recurrent abscess formation.

Nonetheless, given the suspicion for infectious sacroiliitis, a rare cause of back pain in a patient with no risk factors, an MRI was obtained and confirmed left-sided sacroiliitis with a $7.3 \mathrm{~cm}$ abscess extending along the left piriformis (Figure 1).

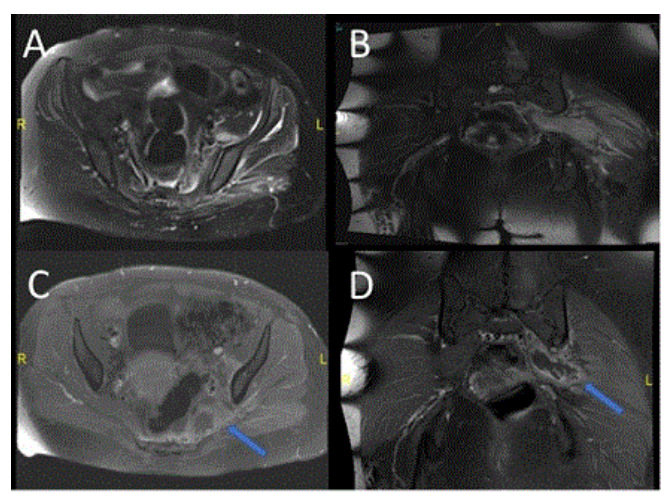

Figure 1: First MRI from the emergency room demonstrating leftsided piriformis abscess and early changes around the left sacroiliac joint. (A) T2 axial view demonstrating fluid within the left sacroiliac joint. (B) T2 coronal view demonstrating edema along the left piriformis muscle and adjacent musculatures. (C, D) post contrast axial and coronal images demonstrating peripheral enhancing piriformis abscess (arrows).

The patient was admitted for interventional radiology-guided drainage of the piriformis abscess and was found to methicillinsusceptible Staphylococcus aureus (MSSA) bacteremia.

Intravenous (IV) antibiotics were narrowed to IV nafcillin, however our patient continued to have increasing left hip-back pain after drainage. 
A repeat MRI (13 days after admission) revealed worsening of sacroiliac joint infection but improvement of the piriformis abscess.

Consequently, worsening sacroiliitis, septic arthritis, and possible osteomyelitis of her left sacrum and iliac bones could not be excluded, and we were challenged to determine the best management option for our patient; surgical intervention versus medical management (Figures $2-4)$.

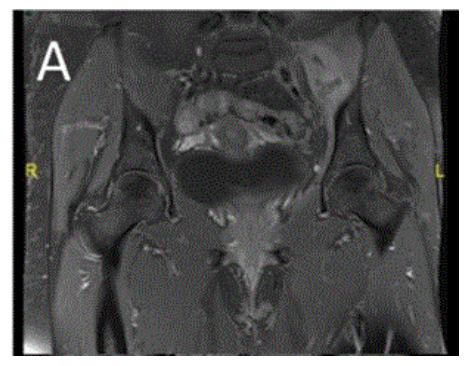

Figure 2: T1 post-contrast coronal view demonstrating progress from treatment with much resolution of the piriformis abscess but continued changes in the sacroiliac joint. Slice is shown at 13 days (d) post-admission.

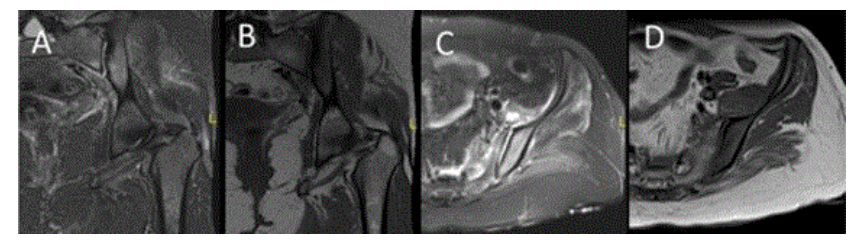

Figure 3: Changes over time around the left sacroiliac joint is shown with MRI sequences in the coronal and axial planes. Columns from left to right show STIR coronal, T1 coronal, T2 fat saturated axial, and $\mathrm{T} 1$ axial views from 13 days (d) post-admission.

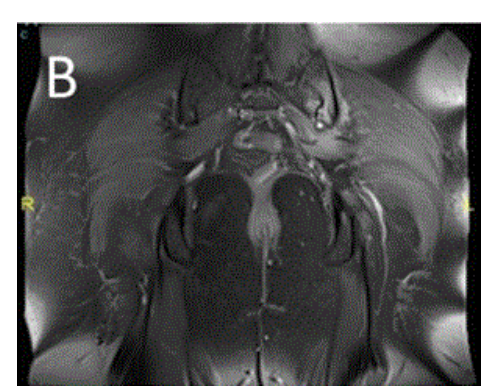

Figure 4: T1 post-contrast coronal view demonstrating progress from treatment with much resolution of the piriformis abscess but continued changes in the sacroiliac joint. Slice is shown at 20 days (d) post-admission.

A course of six weeks of IV nafcillin was selected and another repeat MRI (20 days after admission) demonstrated unchanged sacroiliac joint infection (Figures 2-5).

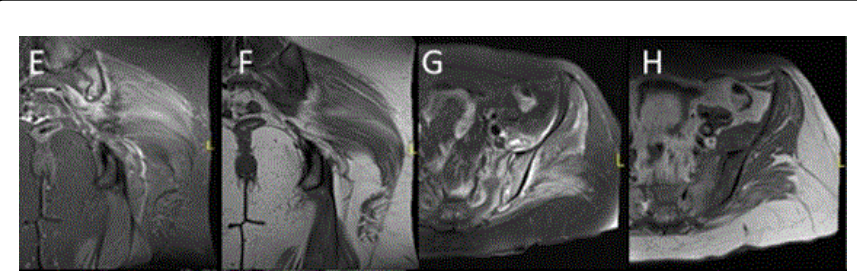

Figure 5: Changes over time around the left sacroiliac joint is shown with MRI sequences in the coronal and axial planes. Columns from left to right show STIR coronal, T1 coronal, T2 fat saturated axial, and $\mathrm{T} 1$ axial views from 20 days (d) post-admission. Erosive changes and marrow edema plateaued around $20 \mathrm{~d}$ post-admission $(\mathrm{E}-\mathrm{H})$.

However, our patient's reported pain score and inflammatory markers had significantly decreased and she was able to ambulate with a rolling walker prior to discharge. On multiple follow up visits, our patient continued to improve clinically, and continued to have downtrending ESR and CRP, and achieving nearly baseline functional status.

In contrast, a follow up MRI (76 days after admission) demonstrated resolution of previously seen abscesses and interval improvement in the sacroiliac joint fluid, yet still with largely unchanged sacroiliac joint appearance relative to previous imaging (Figures 6 and 7).

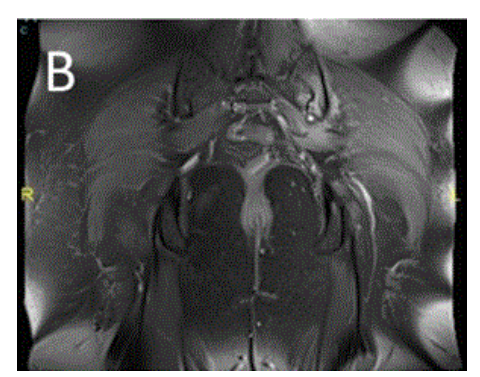

Figure 6: THRIVE post-contrast coronal view demonstrating progress from treatment with much resolution of the piriformis abscess but continued changes in the sacroiliac joint. Slice is shown at 76 days (d) post-admission.

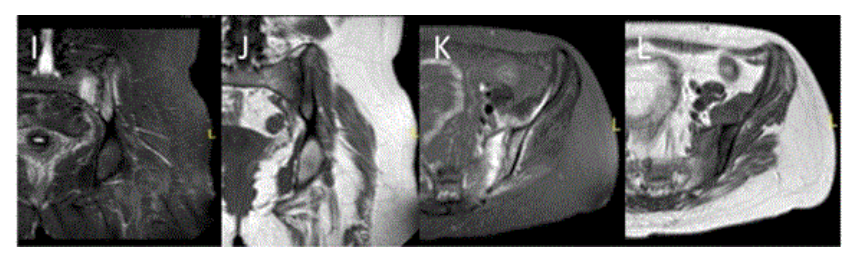

Figure 7: Changes over time around the left sacroiliac joint is shown with MRI sequences in the coronal and axial planes. Columns from left to right show STIR coronal, T1 coronal, T2 fat saturated axial, and $\mathrm{T} 1$ axial views from 76 days (d) post-admission. Edema in the surrounding musculature nearly completely resolved by $76 \mathrm{~d}$ postadmission (I-L). 


\section{Discussion}

Infectious sacroiliitis (ISI) is a frequently delayed or misdiagnosed etiology of sacroiliac joint pain secondary to its vague and nonspecific symptoms. Differential diagnosis for ISI includes inflammatory and degenerative etiologies, such as ankylosing spondylitis, reactive arthritis, psoriatic arthritis, osteoarthritis of the hip, as well as mimics of sacroiliac disease including superior gluteal nerve and obturator nerve entrapments, lumbar disc herniation, discitis, pelvic abscess, and strain injuries. Thus, physical exam alone cannot effectively identify ISI.

The underlying pathophysiology of ISI is thought to involve hematogenous spread of bacteria or local extension of adjacent soft tissue or bone infection [1]. Staphylococcus aureus is the most commonly identified culprit, however Salmonella, Streptococcus, Brucella, Mycobacterium tuberculosis and Pseudomonas can be responsible as well. This mechanism in theory implicates trauma, immunosuppression, rheumatoid arthritis, intravenous drug use, concomitant infection, splenectomy, and pregnancy as potential risk factors. Although our patient did not exhibit any of these risk factors, Vyskocil et al. noted in a review of 166 cases of septic sacroiliitis that $41 \%$ of patients demonstrated no associated factors [3].

Considering our patient's level of acute disability and rapid progression of hip-buttock pain in the setting of unrevealing radiographs, we were still concerned for ISI despite the lack of risk factors and subsequently obtained an MRI of the sacroiliac joint. Radiographic diagnosis of ISI can be difficult in the emergency room on an initial evaluation. Plain radiographs and computed tomography may reveal widening of the joint space and erosive changes of the subchondral plate in longstanding disease, but are frequently normal early in this disease process and may take years to develop. $99 \mathrm{~m}$ technetium-labeled bone scintigraphy is still occasionally in use today due to its low cost, but its diagnostic advantages are only modest when compared to MRI (positive likelihood ratio 3.4 versus 10) and does not deliver spatial resolution and high soft tissue contrast [4-6]. Therefore, MRI remains the gold standard for diagnosis of sacroiliitis. A consensus in the literature defines the following MRI findings necessary to diagnose sacroiliitis: active inflammatory lesions of sacroiliac joints, bone marrow edema on STIR or osteitis on T1 postGadolinium, and sole presence of other active inflammatory lesions such as synovitis, enthesitis, or capsulitis without bone marrow edema $[7,8]$. One study directly compared different imaging modalities in six cases of ISI and found all six cases had positive MRI studies, whereas only three cases had positive computerized tomography (CT) scans studies and only one case had a positive bone scan study [9].

However, to date there are no widely accepted guidelines that define the utility of a follow-up MRI to monitor resolution or progression of ISI in acute or subacute settings. There is only limited evidence suggesting use of diffusion weighted imaging (DWI) in relation to inflammatory markers to assess the therapeutic responses in patients receiving antibiotic treatment for sacroiliitis secondary to Brucella infection [10,11]. Nonetheless, after drainage of our patient's $7.3 \mathrm{~cm}$ piriformis abscess which did not result in significant pain relief, we opted to reimage our patient with an MRI which revealed marked interval worsening of her SI joint. These findings suggested failed medial management and prompted us to consider invasive surgical interventions.

With a multidisciplinary approach, including infectious disease, orthopedic surgery and radiology experts we discussed the risks and benefits of hip-destabilization surgery as well as the lack of evidence for the utility of MRI in monitoring progression of infectious sacroiliitis. As a result, we felt surgical intervention was not indicated and we continued with medical management, with a plan to reimage the SI joint one more time to evaluate for interval changes. Again, on repeat MRI we discovered interval worsening of the SI joint. However, our patient had clinically improved and could ambulate with a rolling walker, bear weight on her left leg, and perform log roll bilaterally without pain. Additionally, inflammatory makers, including CRP, an acute-phase reactant protein released in response to injury and inflammation, were down-trending. In the setting of clinical improvement and decreasing inflammatory biomarkers, surgical intervention was rejected. Our patient was discharged on a 6-week total course of IV nafcillin and close outpatient follow up. A repeat outpatient MRI showed no significant interval change, yet incongruently our patient demonstrated tremendous clinical improvement. We posit that radiographic resolution of sacroiliitis is substantially delayed in relation to clinical improvement, and radiographic findings alone can misguide the clinician to elect an invasive management strategy.

Finally, there is no consensus in duration of antibiotic treatment for infectious sacroiliitis. Select groups have reported lengths of antibiotic therapy anywhere from 28 days to 102 days depending on the infectious etiology of ISI [12-15]. We opted for 6 weeks of IV antibiotics initially and most sources agree 4-weeks of antibiotic therapy is a minimum treatment course for ISI. Broad spectrum antibiotics covering gram-positive bacteria are recommended in the absence of cultures to prevent complications such as abscesses, osteomyelitis, and sepsis, although ideally cultures should be obtained prior to antibiotics are initiated. Furthermore, untreated or delayed diagnosis of ISI has commonly reported complications including recurrent disease, arthritis, chronic pain, and sepsis.

\section{Conclusions: Lessons for the Clinician}

This case serves to emphasize that the clinician must maintain a high index of suspicion for initial evaluation of worsening acute onset unilateral back-hip pain in an otherwise healthy individual, and utilize MR imaging early on to help establish the diagnosis. Next, we advocate both trend in inflammatory biomarkers and serial physical exams should be considered prior to committing to surgical intervention (i.e. joint fusion-stabilization and debridement), rather than radiographic findings alone, which may trail clinical improvement. Only in patients whose pain and inflammatory markers continue to persist despite adequate medical therapy should surgical interventions be offered.

\section{References}

1. Mancarella L, De Santis M, Magarelli N, Ierardi AM, Bonomo L, et al. (2009) Septic sacroiliitis: An uncommon septic arthritis. Clin Exp Rheumatol 27: 1004-1008.

2. Stuber KJ (2007) Specificity, sensitivity, and predictive values of clinical tests of the sacroiliac joint: A systematic review of the literature. J Can Chiropr Assoc 51: 30-41.

3. Vyskocil JJ, McIlroy MA, Brennan TA, Wilson FM (1991) Pyogenic Infection of the Sacroiliac Joint Case Reports and Review of the Literature. Medicine 70: 188-197.

4. Rudwaleit M (2004) How to diagnose axial spondyloarthritis early. Ann Rheum Dis 63: 535-543.

5. Song IH, Brandt H, Rudwaleit M, Sieper J (2010) Limited diagnostic value of unilateral sacroiliitis in scintigraphy in assessing axial spondyloarthritis. J Rheumatol 37: 1200-1202. 
Citation: Malhotra A, Kalil R, Jones R, Schwartz D, Qadeer AH, et al. (2017) Infectious Sacroiliitis: A Radiographic No Touch Lesion. J Gen Pract

Page 4 of 4

6. Sturzenbecher A (2000) MR imaging of septic sacroiliitis. Skeletal Radiol 29: 439-446.

7. Lambert RG, Bakker PC, van der Heijde D, Weber U, Rudwaleit M, et al (2016) Defining active sacroiliitis on MRI for classification of axial spondyloarthritis: Update by the ASAS MRI working group. Ann Rheum Dis 75: 1958-1963.

8. Rudwaleit M, Jurik AG, Hermann KGA, Landewé R, van der Heijde D, et al. (1991) Defining active sacroiliitis on magnetic resonance imaging (MRI) for classification of axial spondyloarthritis: A consensual approach by the ASAS/OMERACT MRI group. Ann Rheum Dis 68: 1520-1527.

9. Klein MA (1991) MR imaging of septic sacroiliitis. J Comput Assist Tomogr 15: 126-132.

10. Bilgeturk A, Gul HC, Karakas A, Mert G, Artuk C, et al. (2017) Can imaging modalities be used as follow-up criteria after brucellar sacroiliitis treatment? J Infect Develop Count 11: 123-128.
11. Sahin N, Hacibeyoglu H, Ince O, Solak A, Uyar B, et al. (2015) Is there a role for DWI in the diagnosis of sacroiliitis based on ASAS criteria? Int J Clin Exp Med 8: 7544.

12. Bellazreg F, Alaya Z, Hattab Z, Lasfar NB, Ayeche ML, et al. (2016) Infectious sacroiliitis in tunisian centre: Retrospective study of 25 cases. Pan Afr Med J 24: 3.

13. Hermet M, Minichiello E, Flipo RM, Dubost JJ, Allanore Y, et al. (2012) Infectious sacroiliitis: A retrospective, multicentre study of 39 adults. BMC Infect Dis 12: 305.

14. Priest JR (2008) Brucellosis and sacroiliitis: A common presentation of an uncommon pathogen. J Am Board Fam Med 21: 158-161.

15. Tseng YC, Yang YS, Wu YC, Chiu SK, Lin TY, et al. (2014) Infectious sacroiliitis caused by Staphylococcus aureus following acupuncture: A case report. Acupunct Med 32: 77-80. 